\title{
Genetic and molecular characterization of myelodysplastic syndromes and related myeloid neoplasms
}

\author{
Bhumika Patel • Cassandra Hirsch • Michael Clemente • \\ Mikkael Sekeres $\cdot$ Hideki Makishima $\cdot$ \\ Jaroslaw P. Maciejewski
}

Received: 15 January 2015 / Accepted: 18 January 2015 / Published online: 18 February 2015

(c) The Japanese Society of Hematology 2015

\begin{abstract}
Whole exome next generation sequencing systematically applied as a discovery tool in myelodysplastic syndromes (MDS) has led to the identification of a large number of novel mutations. Despite hundreds of patients studied, mutational saturation has not been reached and it is expected that new driver mutations will be discovered in this very heterogeneous condition. Serial samples and deep sequencing of the identified alterations has allowed for a dynamic/chronologic analysis of clonal architecture and identification of a subset of ancestral and secondary molecular lesions. Chromosomal gains and losses have been incorporated into the mutational analyses because they can either cooperate with mutations or produce a functional phenocopy. In addition to the search for somatic defects in MDS, similar discovery studies have been also performed to identify germ line mutations/alterations. Clinical analysis showed applicability of multiplexed somatic mutational panels that would complement current pathomorphologic diagnosis, allow for subclassification of nosologic entities, and enhance predictive power of current prognostic algorithms. Overall, comprehensive genomic analysis in
\end{abstract}

B. Patel · C. Hirsch · M. Clemente $\cdot$ M. Sekeres $\cdot$ H. Makishima J. P. Maciejewski

Department of Translational Hematology and Oncology

Research, Taussig Cancer Institute, Cleveland Clinic, Cleveland, $\mathrm{OH}$, USA

M. Sekeres $\cdot$ J. P. Maciejewski

Leukemia Program, Cleveland Clinic Taussig Cancer Institute, Cleveland, OH, USA

J. P. Maciejewski ( $₫)$

Taussig Cancer Institute/R40, 9500 Euclid Avenue, Cleveland, OH 44195, USA

e-mail:maciejj@ccf.org
MDS has revealed a tremendous heterogeneity of somatic lesions and their combinations further enhanced by the heterogeneity of clonal architecture and chromosomal lesions.

Keywords MDS $\cdot$ Somatic mutations $\cdot$ NGS $\cdot$ Clonal architecture $\cdot \mathrm{AML}$

\section{Introduction}

Myelodysplastic syndrome (MDS) is a disease of hematopoietic stem and progenitor cells, characterized by dysplasia, cytopenias and progression to acute myeloid leukemia (AML). Histomorphologic features and the spectrum of genomic alterations in MDS overlap with the closely related MDS/myeloproliferative neoplasms (MDS/MPN) and secondary AML (sAML). Application of new modern high-throughput molecular technologies, including chiefly whole exome sequencing (WES) or targeted multiamplicon next generation sequencing (NGS), along with single nucleotide polymorphisms (SNPs) and comparative genomic hybridization (CGH) arrays in investigation of MDS and related conditions has provided not only a substrate for basic investigations of the pathogenesis of these conditions, but also for conceptualization of new prognostic algorithms and targeted diagnostic tools (Fig. 1). The new diagnostic applications may supplement current prognostic schemes, but also include previously unexplored aspects of clonal burden and discrimination between founder and secondary mutations. Somatic mutations may also guide treatment decisions as to susceptibility or resistance to drugs as well as direct rational drug discovery efforts. 


\section{Sources and implications of molecular heterogeneity of MDS}

The pathomorphologic heterogeneity and variability of clinical courses in MDS are likely a result of a wide spectrum of genetic lesions already discovered to occur in this disease. This diversity is further augmented by the combinatorial variability: one could estimate that on average, around 10 somatic mutations are present in $\operatorname{MDS}[1,2], \mathrm{a}$ figure that is likely to increase by the acquisition of secondary events as the disease progresses. Although it appears that certain combinations are more common, the theoretical potential for diversity is tremendous [3, 4], because not only individual defects, but also their combinations may affect the clinical outcomes [1-4] and obscure simple genotype/phenotype relationships (Fig. 2).

Based on the initial results of sequencing efforts in MDS, it can be estimated that a very high number of cases would be needed to be sequenced to identify all possible mutations present in MDS i.e., to reach saturation so that sequencing of new cases will less likely identify new defects [2, 5]. In particular mutations with a frequency of $<1 \%$ of the whole cohort may require larger number of cases to be sequenced [2]. Notably, such somatic events may have a much higher relative frequency in specific MDS variants, comprising only subfractions
Fig. 1 Research and diagnostic applications of next generation sequencing platforms
Fig. 2 Levels of molecular heterogeneity of MDS

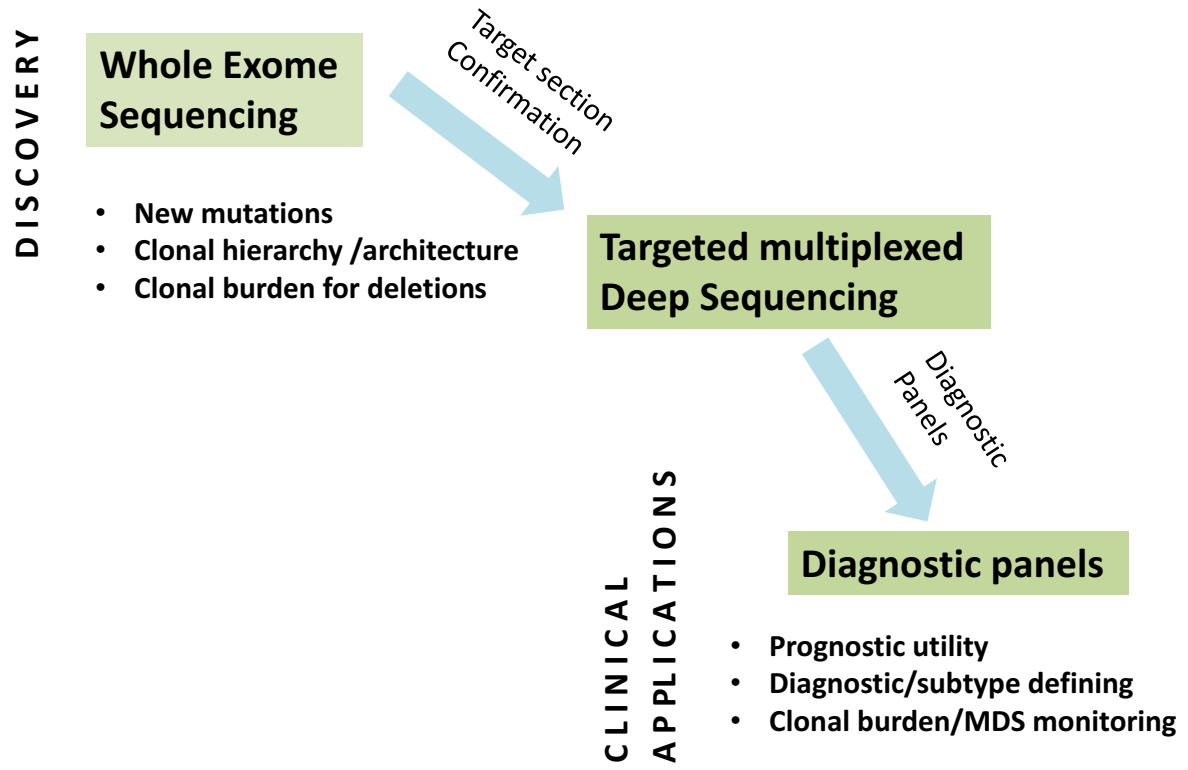


of the unselected cohorts. Moreover, while some genes are affected by canonical mutations, other genes harbor various types of mutations (missense, nonsense, or stop codons) at diverse positions and configurations (heterozygous, homozygous, hemizygous) [6-8]. Consequently, their impact on the resultant clinical phenotype may vary. As a practical conclusion, the molecular diversity may in some instances preclude identification of mutations with a prognostic or diagnostic value and thus proper analysis may need to take under consideration of the molecular diversity of defects within one gene.

The source of heterogeneity of MDS is not only found in somatic changes, but also in the genetic makeup determined by the germ line (GL), including GL-encoded copy number variants (CNV) and SNPs. Many important directly leukemogenic lesions have been identified in childhood leukemias and MDS [9], but the disease anticipation and latency varies and thus, some of them may lead to leukemias in adults (Fig 2). For instance, familial RUNXI mutations present with thrombocytopenia and progress to MDS/AML [10]. Similarly, GL CEBPA or GATA2 mutations have been associated with AML and MDS [11, 12]. In some cases such hereditary mutations manifest in relatively late occurring leukemias such as recently demonstrated for DDX41 mutations [13]. Clearly, there are many other weaker leukemogenic lesions which may constitute a risk factor or lead to leukemia with a very long latency, which precludes their identification as hereditary factors.

In addition to the impact of individual molecular lesions, in combination they may interact with each other's epigenetic changes and GL-encoded variants. The latter non-clonal variants interact with somatic defects including deletions (loss of major protective allele) and somatic uniparental disomy (UDP, leading to duplication of disease prone alleles) [14]. Somatic mutations may occur as missense, nonsense or stop codons and they may be heterozygous, hemizygous through deletions, or through UPD homozygous. Deletions may lead to loss of silencing or gain of silencing (hemi-methylation; Fig. 2).

\section{New molecular defects and their classes}

In addition to activating leukemogenic mutations and gene amplications through duplication of chromosomal material, deletions of chromosomal material, hypomorphic/inactivating mutations; aberrant promoter silencing of tumor suppressor genes (TSG) have been recognized as important pathogenic mechanisms in MDS and other myeloid malignancies. Many molecular defects acquired by the transformed leukemic cell ultimately lead to TSG haploinsufficiency with all the inherent downstream consequences that result in a clonal advantage and progressive malignant evolution. While many initial discoveries of important mutations have been made by deductive approaches, including combination of SNP arrays and Sanger sequencing (e.g., TET2, EZH2, CBL, UTX) [7, 8, 15-18] NGS accelerated the discovery of novel recurrent somatic mutations, a work that was initially pioneered in AML $[19,20]$. Newly discovered classes of somatic defects involve mutations in many members of spliceosomal genes (of which some are strongly related specific morphologic phenotypes), cohesin family of mutations, RNA helicases, mutations involving chromatin-modifying complexes such as PRC2 (e.g., EED, SUZ12 or EZH2), RAS family (e.g., K/N/HRAS, RIT1, PTPN11, etc.), DNA histone demethylases and methylases, synergistically acting signal tranducers, transcription factors, new kinases and growth factor receptors $(C$ KIT, $C-M P L, C S F 3 R$, etc.) or genes coding for metabolic enzymes, among others. Similar grouping can be made for some of the germ line lesions for example FA genes, telomerase machinery genes, and microsatellite instability. Combining mutational events in individual genes into the functional classes $[4,21]$ while rational may not reflect the biology and obscure existing clinical and pathomorphologic associations. For instance, TET2 mutations are often grouped functionally with $I D H 1 / 2$ mutations, yet these mutations are present in prognostically distinct subtypes of MDS and the functional impact on phenotype is not firmly established. Similarly, spliceosomal mutations are often grouped for analytic purposes yet they may affect spliceosomal machinery at different stages of splicing process and therefore may have unrelated downstream consequences as reflected by often distinct phenotypes. Even functionally grouped genes may not be reflective of resultant impact, for example homozygous deleterious changes may be much more permissive than missense changes e.g., in positions within the gene peripheral to acting sites. In addition, the impact of mutations may depend on their position within clonal hierarchy (see below ancestral vs. secondary lesions, intratumor heterogeneity, and clonal hierarchy).

Another approach to better comprehend the molecular diversity is to combine loss-of-function mutant cases with those which harbor corresponding deletions or conversely gain of function mutations with corresponding microamplifications. It is conceivable that various molecular lesions can produce phenocopy and also similar mechanistic consequences. For instance, a TSG haploinsuffciency may be caused by diverse mechanisms, such as promoter hypermethylation, deletion, hypomorphic mutations and missplicing due to a specific spliceosomal mutation. Recognition of such coherent mechanisms may be important for future molecular characterization of individual cases and subclassification based on functionally convergent mutations (Fig. 3). 
Fig. 3 Functional convergence of pathogenic pathways consequent to the diverse mutations

Fig. 4 Implications of clonal architecture and hierarchy

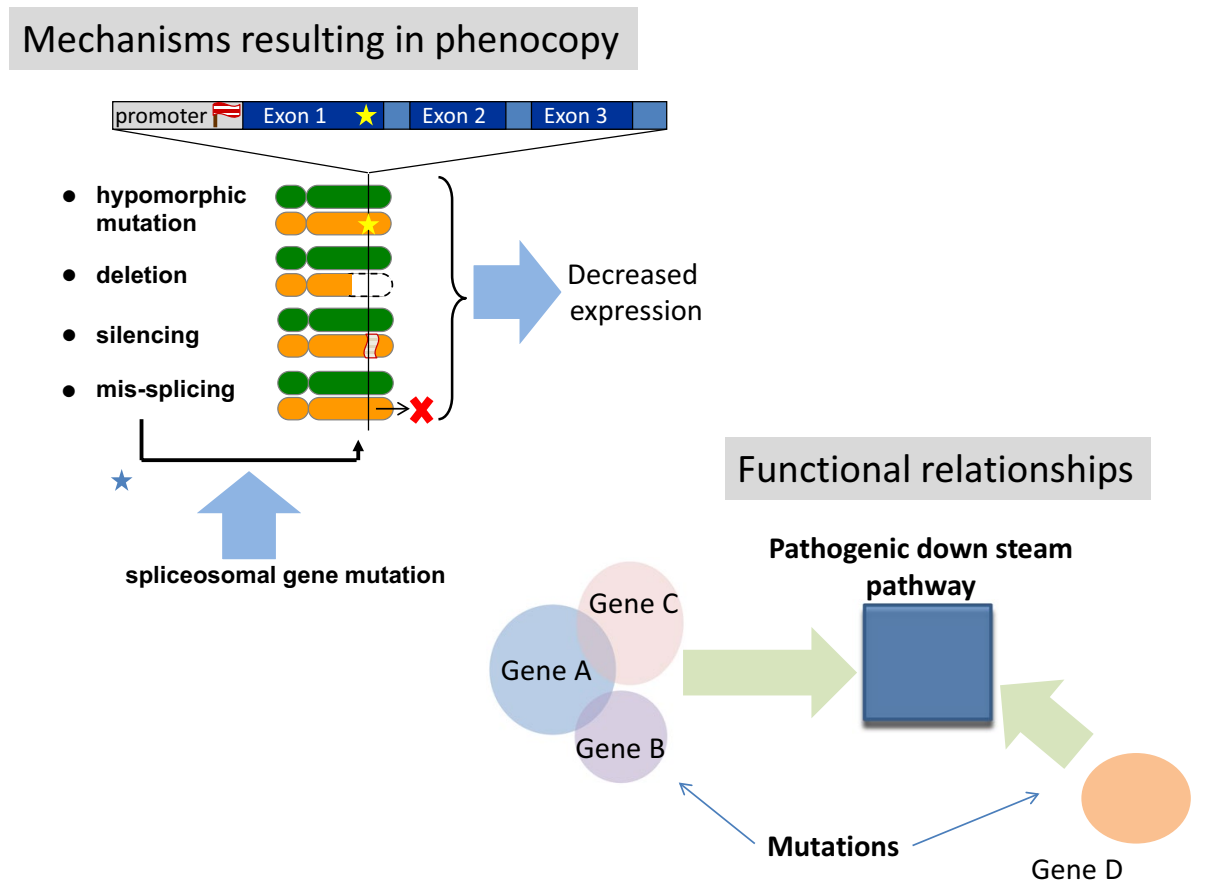

Calculation by VAF and copy number

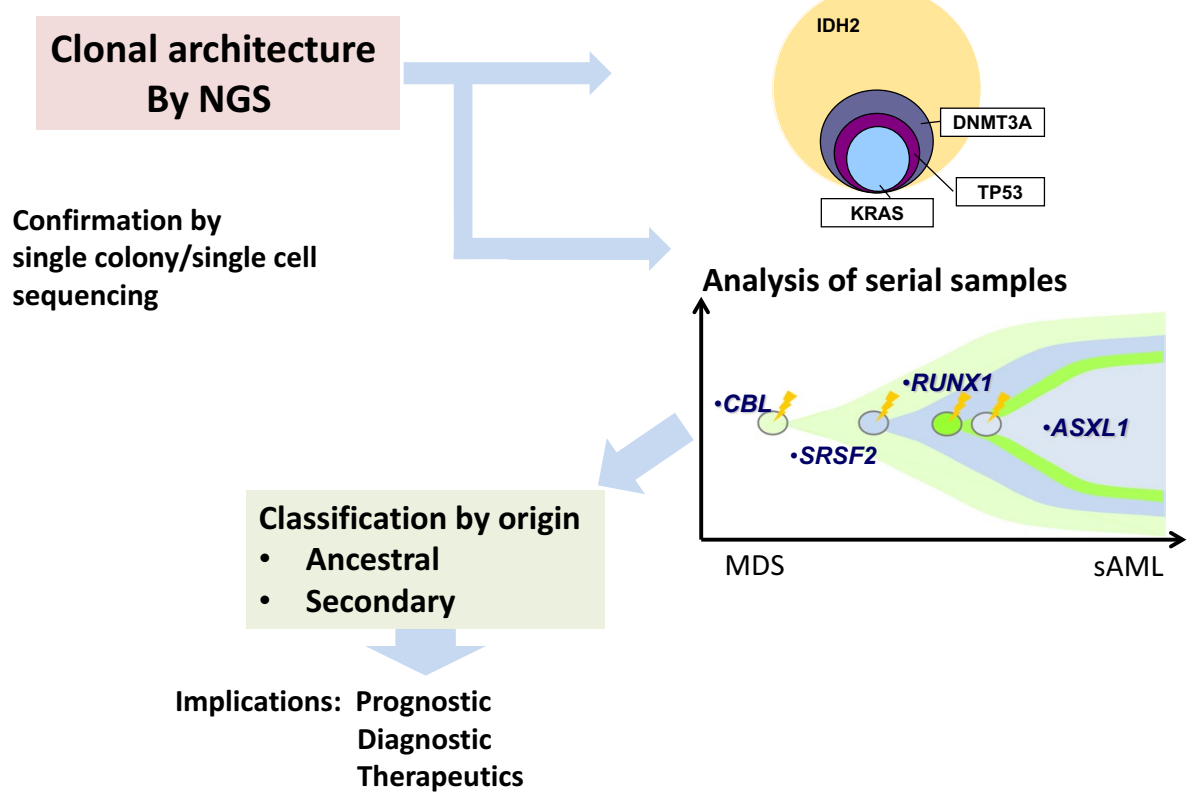

\section{Intratumor heterogeneity and clonal hierarchy}

One of the major conclusions of NGS studies was recognition of intratumor heterogeneity and complex hierarchical clonal structure in leukemia [22, 23] including MDS (Fig. 4). While theoretically the simple clonal burden (or clonal size) may have impact on outcomes, to date major differences have not been identified, implying that the sheer presence of an aggressive mutation may be predictive of poor outcome irrespective of the clonal burden. However, these aspects have not been sufficiently explored leaving the issues of significance of tiny clones at the threshold of sensitivity levels of deep NGS to be further investigated. To that end deep sequencing provides a higher sensitivity for detection of smaller clones and thus, would have greater sensitivity in detection of specific mutations than Sanger's sequencing or WES. Overall by calculating the clonal burden one can follow therapy responses and define them on 
a molecular level; this application may play a future role in monitoring of therapy responses and assessment of the quality of response including the presence of minimal residual disease (MRD). However, clonal burden may be more important when evaluated in conjunction with other mutations. Clearly, an ancestral mutation would be present in all clonal cells while secondary mutation would have smaller clonal size and be therefore subclonal. While using only the variant allelic frequency may be not informative in terms of clonal composition as in smaller clones may be a result of clonal mosaicism rather than subclonal evolution. Similarly, mutations with similar VAFs may be found for 2 different mutations, the issue which is the primary event may not be statistically resolvable. However, in most cases the hierarchical analysis can be performed and when serial samples are available, the clonal kinetics is assessed. In general, for the proper assignment of ancestral events WES may be followed by deep NGS. To calculate the clonal burden, the copy number information e.g., from SNP arrays is needed.

Of note is that in many ways the sheer size of the clone is not the most important, but rather clonal ranking with the largest clone is suggestive of being the ancestral event. Growing evidence suggests that it is not the size of the clone, but the position within clonal hierarchy which is the most relevant parameter with ancestral mutations being most clinically relevant. Early results demonstrate that ancestral vs. secondary events affecting the same gene seem to have a distinct impact on clinical phenotypes and outcomes.

Deep sequencing allows for very sensitive detection of mutations in a targeted fashion. This property may account for the differences in the perceived distribution of somatic events analysed by various methods. Sanger's sequencing and even WES may not detect smaller clones which would be detectable by deep sequencing and would be most suitable for disease monitoring. However, when paired WES is applied to tumor and GL DNA, mathematical calculations based on comparison of VAF in tumor vs. GL for informative heterozygous SNPs allow for calculation of clonal burden for deletions.

\section{Molecular characterization of MDS: mutational landscape and its implication for diagnosis and prognostication}

We have applied WES to define the landscape of mutational events in MDS based on analysis of a large number of exomes and cohort of patients. We have identified topmost frequent mutations that have been targeted using multiamplicon deep NGS panel $[1,2]$. While, others have mostly selected gene panels and used them for analysis of large cohorts of patients $[3,4]$. In terms of the distribution, the results of the most common mutations are comparable, but clearly the composition of the screening cohort used in these studies will affect frequencies of certain mutations. For instance, various cohorts showed different frequencies of SF3B1 or TET2 mutations depending on the proportion of RARS or CMML cases included in these cohorts $[6,7,24,25]$. Other differences may be due to the depth of sequencing and ability to evenly cover all gene areas included in the panels. Moreover, most of the targeted panels do not include GL controls and apart of known pathogenetic and canonical mutations; many of the reported mutations have not been confirmed. For instance in a recent study [3], a high frequency of MLL3 mutations have been reported, but it is known from confirmatory studies that most of these defects cannot be validated.

Emerging evidence demonstrates that there is a great deal of overlap in the mutational spectrum between AML (in particular non-core binding factor AML) and MDS, but also many significant differences. Similarities and distinctive difference are also present when MDS/MPN and MDS are compared [1,2], for some typical ancestral event may herald molecular/pathogenetic relation to other morphologically defined subentities. While so far an exact resolution between classical subentities has not been successful when morphology is used as gold standard, independent molecular classification schemes may be ultimately more reflective of molecular pathogenesis and similarity.

Unbiased screens showed the presence of new families of genes affected by mutations or new members of gene families known to be affected by mutations. MDS subtypes can be characterized by distribution of most common mutations or by the presence of specific ancestral events, the later approach allows to compare the clinical outcomes of cases with different ancestral mutations because per definition each case is only characterized only by one mutation. In contrast, when all mutations are used for molecular classification comparisons can be made only between mutant and WT cases as each patient may have multiple mutations. For diagnostic application targeted panels of mutations may be used, in particular including lesions which have shown prognostic significance. Among many mutations found so far prognostic impact has been found only for minority of them, but clearly this is a topic open for further study. Lack of clear clinical impact may be due the presence of various mutations and chromosomal aberration combinations or simply due to lack of statistical power for many of the defects present only in a subfraction of patients. Similarly, it may be difficult to demonstrate the impact on outcomes for many of the secondary mutations because the prognosis of advanced, high-risk cases is already very poor. Future analyses may involve groups of functionally related mutations or as described above analysis of clonal architecture 
and correlation of clinical parameters only with founder lesions. For instance, only $60 \%$ of TET2 mutations and $80 \%$ of DNMT3A and thus one could envision analysis involving only founder mutations [2].

\section{Conclusions}

In sum, the field of leukemia genetics is transitioning from discovery phase to application phase. While many of the newly discovered lesions may constitute targets for rational drug discovery, the first translation of the research results will be in diagnostics. Targeted rationally selected panels that include known lesions and contain information about clonal burden will find increased application as routine diagnostic tools. Initial application will allow for better assessment of prognosis or distinction of clonal process in ambiguous cases but will also be used for monitoring of MRD or rational drug applications.

\section{References}

1. Makishima H, LaFramboise T, Przychodzen BP, Yoshida K, Ruffalo M, Ines Gomez-Segui I, et al. Clinical MUTATOME of myelodysplastic syndrome; comparison to primary acute myelogenous leukemia. Blood (ASH Annual Meeting Abstracts). 2013;122:518.

2. Makishima H, Yoshida K, LaFramboise T, Przychodzen BP, Ruffalo M, Ines Gomez-Segui I, et al. Landscape of somatic mutations in whole exomes of myelodysplastic syndromes and related myeloid neoplasms. Nat Genet. 2014 (submission).

3. Papaemmanuil E, Gerstung M, Malcovati L, Tauro S, Gundem G, Van Loo P, et al. Clinical and biological implications of driver mutations in myelodysplastic syndromes. Blood. 2013;122:3616-27.

4. Haferlach T, Nagata Y, Grossmann V, Okuno Y, Bacher U, Nagae $\mathrm{G}$, et al. Landscape of genetic lesions in 944 patients with myelodysplastic syndromes. Leukemia. 2014;28:241-7.

5. Lawrence MS, Stojanov P, Mermel CH, Robinson JT, Garraway LA, Golub TR, et al. Discovery and saturation analysis of cancer genes across 21 tumour types. Nature. 2014;505:495-501.

6. Delhommeau F, Dupont S, Della Valle V, James C, Trannoy S, Masse A, et al. Mutation in TET2 in myeloid cancers. N Engl J Med. 2009;360:2289-301.

7. Jankowska AM, Szpurka H, Tiu RV, Makishima H, Afable M, Huh J, et al. Loss of heterozygosity $4 \mathrm{q} 24$ and TET2 mutations associated with myelodysplastic/myeloproliferative neoplasms. Blood. 2009;113:6403-10.

8. Jankowska AM, Makishima H, Tiu RV, Szpurka H, Huang Y, Traina F, et al. Mutational spectrum analysis of chronic myelomonocytic leukemia includes genes associated with epigenetic regulation: UTX, EZH2, and DNMT3A. Blood. 2011;118:3932-41.

9. Stieglitz E, Loh ML. Genetic predispositions to childhood leukemia. Ther Adv Hematol. 2013;4:270-90.

10. Owen CJ, Toze CL, Koochin A, Forrest DL, Smith CA, Stevens JM, et al. Five new pedigrees with inherited RUNX1 mutations causing familial platelet disorder with propensity to myeloid malignancy. Blood. 2008;112:4639-45.

11. Owen C, Barnett M, Fitzgibbon J. Familial myelodysplasia and acute myeloid leukaemia - a review. $\mathrm{Br} \mathrm{J}$ Haematol. 2008; 140:123-32.

12. Hahn CN, Chong CE, Carmichael CL, Wilkins EJ, Brautigan PJ, Li XC, et al. Heritable GATA2 mutations associated with familial myelodysplastic syndrome and acute myeloid leukemia. Nat Genet. 2011;43:1012-7.

13. Polprasert C, Isabell Schulze I, Sekeres MA, Makishima H, Przychodzen B, Hosono N, et al. Novel pathogenic defects of DEAD/H-box helicases in myeloid neoplasms. Cancer Cell. 2014 (revised).

14. Makishima H, Maciejewski JP. Pathogenesis and consequences of uniparental disomy in cancer. Clin Cancer Res. 2011;17:3913-23.

15. Ernst T, Chase AJ, Score J, Hidalgo-Curtis CE, Bryant C, Jones $\mathrm{AV}$, et al. Inactivating mutations of the histone methyltransferase gene EZH2 in myeloid disorders. Nat Genet. 2010;42:722-6.

16. Makishima H, Jankowska AM, Tiu RV, Szpurka H, Sugimoto Y, $\mathrm{Hu} \mathrm{Z}$, et al. Novel homo- and hemizygous mutations in EZH2 in myeloid malignancies. Leukemia. 2010;24:1799-804.

17. Sanada M, Suzuki T, Shih LY, Otsu M, Kato M, Yamazaki S, et al. Gain-of-function of mutated C-CBL tumour suppressor in myeloid neoplasms. Nature. 2009;460:904-8.

18. Makishima H, Cazzolli H, Szpurka H, Dunbar A, Tiu R, Huh J, et al. Mutations of e3 ubiquitin ligase cbl family members constitute a novel common pathogenic lesion in myeloid malignancies. J Clin Oncol. 2009;27:6109-16.

19. Ley TJ, Ding L, Walter MJ, McLellan MD, Lamprecht T, Larson DE, et al. DNMT3A mutations in acute myeloid leukemia. $\mathrm{N}$ Engl J Med. 2010;363:2424-33.

20. Mardis ER, Ding L, Dooling DJ, Larson DE, McLellan MD, Chen $\mathrm{K}$, et al. Recurring mutations found by sequencing an acute myeloid leukemia genome. N Engl J Med. 2009;361:1058-66.

21. Cancer Genome Atlas Research Network. Genomic and epigenomic landscapes of adult de novo acute myeloid leukemia. $\mathrm{N}$ Engl J Med. 2013;368:2059-74.

22. Walter MJ, Shen D, Ding L, Shao J, Koboldt DC, Chen K, et al. Clonal architecture of secondary acute myeloid leukemia. N Engl J Med. 2012;366:1090-8.

23. Walter MJ, Shen D, Shao J, Ding L, White BS, Kandoth C, et al. Clonal diversity of recurrently mutated genes in myelodysplastic syndromes. Leukemia. 2013;27:1275-82.

24. Papaemmanuil E, Cazzola M, Boultwood J, Malcovati L, Vyas P, Bowen D, et al. Somatic SF3B1 mutation in myelodysplasia with ring sideroblasts. N Engl J Med. 2011;365:1384-95.

25. Makishima H, Visconte V, Sakaguchi H, Jankowska AM, Abu Kar S, Jerez A, et al. Mutations in the spliceosome machinery, a novel and ubiquitous pathway in leukemogenesis. Blood. 2012;119:3203-10. 